\title{
openheart Downstream testing after CT coronary angiography: time for a rethink?
}

Gareth Morgan-Hughes (D) , Michelle Claire Williams (D) , ${ }^{2,3}$ Margaret Loudon (D) ,1 Carl A Roobottom, ${ }^{4}$ Alice Veitch, ${ }^{5}$ Robin Van Lingen, ${ }^{6}$ Ben Holloway, ${ }^{7}$ Nicholas Bellenger, ${ }^{8}$ Matthias Schmitt, ${ }^{9}$ Russel Bull ${ }^{10}$

To cite: Morgan-Hughes G, Williams MC, Loudon M, et al. Downstream testing after CT coronary angiography: time for a rethink?. Open Heart 2021;8:e001597. doi:10.1136/ openhrt-2021-001597

Received 22 January 2021 Revised 7 February 2021 Accepted 8 February 2021

Check for updates

C Author(s) (or their employer(s)) 2021. Re-use permitted under CC BY-NC. No commercial re-use. See rights and permissions. Published by BMJ.

${ }^{1}$ Cardiology, University Hospitals Plymouth NHS Trust, Plymouth, UK

${ }^{2}$ Centre for Cardiovascular Sciences, University of Edinburgh, Edinburgh, UK ${ }^{3}$ Radiology, University of Edinburgh, Edinburgh, UK ${ }^{4}$ Radiology, University Hospitals Plymouth NHS Trust, Plymouth, UK

${ }^{5}$ Radiology, Salisbury Hospital NHS Foundation Trust, Salisbury, UK

${ }^{6}$ Radiology, Royal Cornwall Hospitals NHS Trust, Truro, UK

${ }^{7}$ Radiology, University Hospitals Birmingham NHS Foundation Trust, Birmingham, UK ${ }^{8}$ Cardiology, Royal Devon and Exeter Hospital, Exeter, UK

${ }^{9}$ Cardiology, Manchester University NHS Foundation Trust, Manchester, UK

${ }^{10}$ Radiology, Royal Bournemouth Hospital, Bournemouth, UK

Correspondence to Dr Gareth Morgan-Hughes; garethmh@me.com

\section{ABSTRACT}

Objective We surveyed UK practice and compliance with the National Institute for Health and Care Excellence (NICE) 'recent-onset chest pain' guidance (Clinical Guideline 95, 2016) as a service quality initiative. We aimed to evaluate the diagnostic utility and efficacy of CT coronary angiography (CTCA), NICE-guided investigation compliance, invasive coronary angiography (ICA) use and revascularisation.

Methods A prospective analysis was conducted in nine UK centres between January 2018 and March 2020. The reporter decided whether the CTCA was diagnostic. Coronary artery disease was recorded with the Coronary Artery Disease-Reporting and Data System (CAD-RADS). Local electronic records and picture archiving/communication systems were used to collect data regarding functional testing, ICA and revascularisation. Duplication of coronary angiography without revascularisation was taken as a surrogate for ICA overuse.

Results 5293 patients (mean age, $57 \pm 12$ years; body mass index, $29 \pm 6 \mathrm{~kg} / \mathrm{m}^{2} ; 50 \%$ men) underwent CTCA, with a $96 \%$ diagnostic scan rate. $618(12 \%)$ underwent ICA, of which 48\% (298/618) did not receive revascularisation. 3886 (73\%) had CAD-RADS 0-2, with 1\% (35/3886) undergoing ICA, of which $94 \%(33 / 35)$ received ICA as a second-line test. 547 (10\%) had CAD-RADS 3, with $23 \%$ (125/547) undergoing ICA, of which $88 \%$ (110/125) chose ICA as a second-line test, with $26 \%$ (33/125) leading to revascularisation. For 552 (10\%) CAD-RADS 4 and 91 (2\%) CAD-RADS 5 patients, ICA revascularisation rates were $64 \%(221 / 345)$ and $74 \%(46 / 62)$, respectively.

Conclusions While CTCA for recent-onset chest pain assessment has been shown to be a robust test, which negates the need for further investigation in three-quarters of patients, subsequent ICA overuse remains with almost half of these procedures not leading to revascularisation.

\section{INTRODUCTION}

The 2016 iteration of the National Institute for Health and Care Excellence (NICE) guidance for the assessment and diagnosis of recent-onset chest pain of suspected cardiac origin (Clinical Guideline 95 (CG95)) recommends diagnostic testing when stable angina cannot be excluded by clinical assessment alone. ${ }^{1}$ The first-line diagnostic test suggested

\section{Key questions}

What is already known about this subject?

- The 2016 iteration of the National Institute for Health and Care Excellence (NICE) guidance for the assessment and diagnosis of recent-onset chest pain of suspected cardiac origin (Clinical Guideline 95 (CG95 2016)) is clear in its recommendations regarding non-invasive cardiac imaging tests. What is not known is the diagnostic utility and efficacy of CT coronary angiography (CTCA) used in this context the level of NICE compliance and whether invasive coronary angiography (ICA) is being overused.

What does this study add?

- In routine clinical use, CTCA, for recent-onset chest pain assessment, has been shown to be a robust test, undertaken with gender equality, which negates the need for further investigation in $73 \%$ of patients. However, ICA overuse is suggested by $48 \%$ of patients undergoing ICA but not revascularisation. Only $6 \%$ of patients with a moderate stenosis undergo revascularisation, but ICA was commonly used as second-line investigation in this subgroup contrary to NICE CG95 (2016).

How might this impact on clinical practice?

- Service quality improvement may be achieved by taking the onward revascularisation rate into account when considering the need for onward testing and then following NICE CG95 (2016) second-line investigation recommendations and avoiding 'standalone' diagnostic ICA after CTCA wherever possible. These measures may reduce the number of patients undergoing ICA with no resulting revascularisation.

is CT coronary angiography (CTCA). The guideline then recommends additional diagnostic investigation that may be needed following a CTCA, when evidence of coronary artery disease (CAD) of uncertain functional significance is found or the scan is non-diagnostic. In these circumstances, noninvasive functional imaging for myocardial ischaemia is recommended. The rationale for this is that there is little logic in following one anatomical test with another. Invasive 
coronary angiography (ICA) is therefore reserved as a third-line investigation.

Data from North America suggest that $66 \%$ of patients with moderate coronary stenosis on CTCA undergo ICA within 30 days and that $<10 \%$ of these are both symptomatic and receiving antianginal drug therapy. ${ }^{2}$ In the UK, single-centre data from 2016 showed that after a diagnostic CTCA, $42 \%$ of patients who underwent ICA did not have subsequent revascularisation. ${ }^{3}$ Diagnostic ICA following CTCA, without subsequent revascularisation, may have a heterogeneous aetiology. Nevertheless, it commonly represents unnecessary duplication of anatomical testing and may be taken as a surrogate marker for overuse of ICA. This brings with it attendant concerns over timeliness, cost and safety.

Through collaboration with the British Society of Cardiovascular Imaging/British Society of Cardiovascular Computed Tomography (BSCI/BSCCT), we performed a service quality improvement initiative concerning patients across the UK referred for CTCA, following recent-onset chest pain assessment, according to NICE CG95 (2016). The aims were to evaluate the diagnostic utility and efficacy of CTCA in 'real-world' UK practice, to assess compliance with NICE-guided second-line investigation and to assess the use of ICA and revascularisation. This report of the initiative has been completed with reference to the revised standards for quality improvement reporting excellence template (SQUIRE 2). ${ }^{4}$

\section{METHODS \\ Study design}

This is a multicentre, prospective analysis of the onward investigation and treatment of patients with suspected stable CAD assessed for recent-onset chest pain symptoms with CTCA. Centres across the UK undertaking CTCA in accordance with NICE CG95 (2016) were offered the opportunity to collect prospective data on patients attending for CTCA through email communication from the BSCI/BSCCT. It was a requirement that centres had access to information on subsequent activity for patients. The study was registered as a national audit and service evaluation in Plymouth (National audit of downstream testing after CTCA following NICE CG95 (2016) reference: CA_2017-18-191)). Centres agreeing to collect data registered with their local audit departments. Written informed consent and ethical approval were not obtained as patient care was not affected. Only fully anonymised data were transferred and used in the analysis.

\section{Data collection}

Each site was required to make two data collections: baseline data and follow-up data, within 12 months. Baseline data were collected for consecutive patients, fulfilling NICE CG95 (2016) referral criteria at the time of the CTCA, by 10 centres across the UK between January 2018 and March 2019. Local electronic patient records and picture archiving/communication systems were then used to collect follow-up data, which was completed by March 2020.

The first data collection covered demographic information and CTCA results. Demographic information included age, sex and body mass index (BMI). The reporting cardiologist or radiologist decided whether the CTCA was diagnostic or not, primarily based on their own criteria. For all diagnostic CTCA, the Coronary Artery Disease-Reporting and Data System (CADRADS) score was provided (CAD-RADS $0=0 \%$ coronary stenosis; CAD-RADS $1=1 \%-24 \%$; CAD-RADS $2=25 \%-$ $49 \%$; CAD-RADS $3=50 \%-69 \%$; CAD-RADS $4=70 \%-$ 99\%; CAD-RADS $5=100 \%$ or coronary occlusion), with the most severe stenosis defining the patient's score. ${ }^{56}$ The ability to provide a CAD-RADS score was recommended to reporting centres as a secondary method of defining a diagnostic CTCA. The CAD-RADS score was then categorised into three groups (CAD-RADS 0, normal coronary arteries; CAD-RADS 1-2, atheroma probably not associated with ischaemia; CAD-RADS 3-5, atheroma possibly or probably associated with ischaemia)

The second data collection pertained to the completion of the patient's diagnostic pathway. Once a decision regarding onward investigation or treatment after CTCA had been made, and any functional testing for ischaemia, ICA or revascularisation by means of percutaneous intervention (PCI) or coronary artery bypass surgery (CABG) had been carried out, the data collection was complete. If fractional flow reserve derived from CT (FFRCT; Heartflow, Redwood City, California, USA) was used or functional testing for ischaemia was carried out to facilitate the decision-making process regarding the need for ICA, then this was recorded. If patients presented urgently or emergently while planned investigations were pending and underwent further investigation or treatment, then these data were included in the analysis. Data collection did not allow for re-presentation or reinvestigation of stable chest pain symptoms.

All data were then anonymised at the collecting site and collated in Plymouth at the registered national audit centre. One centre submitted uninterpretable data and was excluded from the analysis, resulting in nine centres contributing.

\section{Statistical analysis}

Data pertaining to the entire population are referred to as total population data, and when patients with nondiagnostic scans have been excluded, they are referred to as the diagnostic population. Patient age and BMI are given in the text as mean $\pm \mathrm{SD}$, and ranges are given in tables. Ordinal categorical variables (CAD-RADS scores) are presented as number (percentage) and graphically displayed in bar charts. Percentage and proportional data are presented in tables. Descriptive statistical analysis was performed using SPSS software statistics V.23 (SPSS). 
Table 1 Patient numbers and characteristics by centre

\begin{tabular}{|c|c|c|c|c|c|c|c|c|c|c|}
\hline Centre & A & B & C & D & $\mathbf{E}$ & $\mathbf{F}$ & G & $\mathbf{H}$ & $\mathbf{I}$ & Overall \\
\hline Number of patients & 1037 & 264 & 666 & 198 & 230 & 355 & 513 & 142 & 1888 & 5293 \\
\hline Mean age in years (range) & $\begin{array}{l}61 \\
(22-91)\end{array}$ & $\begin{array}{l}56 \\
(24-82)\end{array}$ & No data & $\begin{array}{l}51 \\
(17-76)\end{array}$ & $\begin{array}{l}55 \\
(32-76)\end{array}$ & $\begin{array}{l}57 \\
(18-89)\end{array}$ & $\begin{array}{l}59 \\
(25-87)\end{array}$ & $\begin{array}{l}55 \\
(20-84)\end{array}$ & $\begin{array}{l}55 \\
(17-86)\end{array}$ & $\begin{array}{l}57^{*} \\
(17-91)\end{array}$ \\
\hline $\begin{array}{l}\text { Male, } \mathrm{n} \\
(\%)\end{array}$ & $\begin{array}{l}557 \\
(54)\end{array}$ & $\begin{array}{l}99 \\
(38)\end{array}$ & No data & $\begin{array}{l}110 \\
(56)\end{array}$ & $\begin{array}{l}124 \\
(54)\end{array}$ & $\begin{array}{l}187 \\
(53)\end{array}$ & $\begin{array}{l}280 \\
(55)\end{array}$ & $\begin{array}{l}76 \\
(54)\end{array}$ & $\begin{array}{l}893 \\
(47)\end{array}$ & $\begin{array}{l}2326^{*} \\
(50)\end{array}$ \\
\hline Mean BMI $\left(\mathrm{kg} / \mathrm{m}^{2}\right)$ (range) & $\begin{array}{l}29 \\
(15-67)\end{array}$ & $\begin{array}{l}29 \\
(15-60)\end{array}$ & No data & $\begin{array}{l}27 \\
(17-43)\end{array}$ & $\begin{array}{l}30 \\
(19-48)\end{array}$ & $\begin{array}{l}30 \\
(17-61)\end{array}$ & $\begin{array}{l}28 \\
(15-55)\end{array}$ & $\begin{array}{l}30 \\
(18-52)\end{array}$ & $\begin{array}{l}29 \\
(17-55)\end{array}$ & $\begin{array}{l}29 * \\
(15-67)\end{array}$ \\
\hline
\end{tabular}

The overall data therefore refer to eight centres, 4627 patients.

${ }^{*}$ One centre did not submit data pertaining to age and BMI.

BMI, body mass index.

\section{RESULTS}

\section{Patient population}

The total population contained data from 5293 patients. Patients had a mean age of $57 \pm 12$ years, mean BMI of $29 \pm 6 \mathrm{~kg} / \mathrm{m}^{2}$, and $50 \%$ were men and $50 \%$ women (table 1). Overall, $96 \%$ of CTCA were diagnostic.

The CAD-RADS distribution is given in figure 1. For the 5076 diagnostic population, 1976 (39\%) had normal coronary arteries (CAD-RADS 0), 1910 (38\%) had atheroma probably not associated with ischaemia (CADRADS 1-2) and 1190 (23\%) had atheroma possibly or probably associated with ischaemia (CAD-RADS 3-5) (table 2).

\section{Downstream testing}

All patients

Of the total population, $416(8 \%)$ underwent functional testing after CTCA, and of these, $399(96 \%)$ did not undergo subsequent revascularisation. Three centres submitted FFRCT data; however, the total numbers were modest at 208 (4\% of diagnostic population). Of these, $88 \%(183 / 208)$ did not undergo revascularisation, but

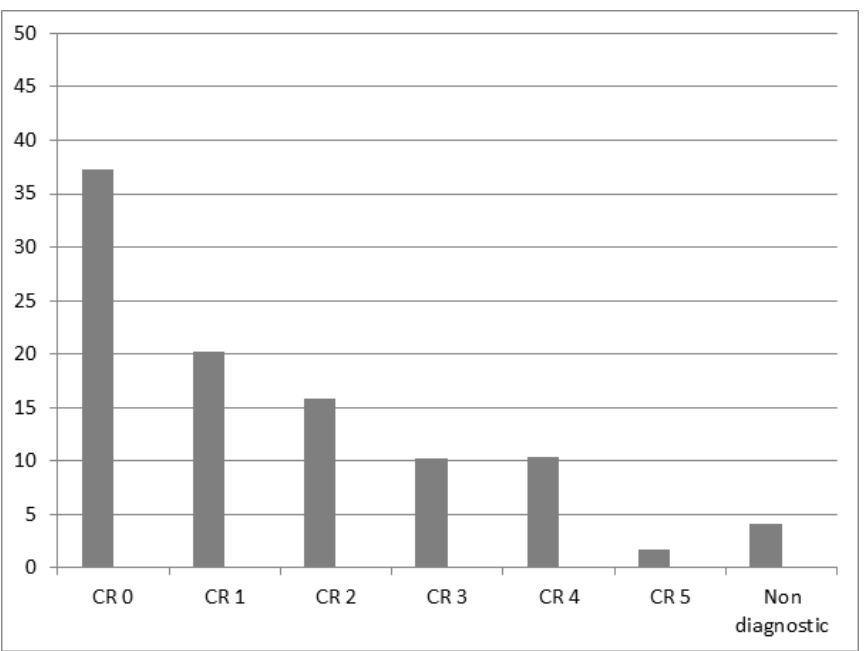

Figure 1 Coronary Artery Disease-Reporting and Data System (CR) distribution (\%) for 5293 patients undergoing CT coronary angiography for chest pain assessment following National Institute for Health and Care Excellence-Clinical Guidance 95 (2016). no other subgroup analysis was made. Of the total population, 618 (12\%) patients underwent ICA, with 298 $(48 \%)$ of these receiving no revascularisation, 242 (39\%) undergoing PCI and 78 (13\%) undergoing CABG.

\section{CAD-RADS 0 and CAD-RADS 1-2}

Of the 1976 patients with normal coronary arteries (CADRADS 0), 39 (2\%) underwent functional testing (functional testing by CAD-RADS score is shown in figure 2) and 3 underwent ICA (ICA by CAD-RADS score is shown in figure 3) with no revascularisation.

Of the 1910 patients with atheroma probably not associated with ischaemia (CAD-RADS 1-2), functional imaging was used in $41(2 \%)$ and ICA was used in $32(2 \%)$. Two CAD-RADS 2 patients underwent PCI (figure 4).

In total, there were 3886 patients with normal coronary arteries (CAD-RADS 0 ) or atheroma probably not associated with ischaemia (CAD-RADS 1-2). According to NICE CG95 (2016), this means that $77 \%$ of the diagnostic population and $73 \%$ of the total population would not need second-line investigation.

From both groups (CAD-RADS 0 and CAD-RADS 1-2), $33(94 \%)$ of 35 patients who underwent ICA did not undergo revascularisation (figure 5).

\section{CAD-RADS 3-5}

The diagnostic population with CAD-RADS 3,4 or 5 consisted of 1190 patients (23\%). For these patients, functional testing for ischaemia (figure 2) was used in 165 (30\%) CAD-RADS 3 patients, 114 (21\%) CAD-RADS 4 patients and $8(9 \%)$ CAD-RADS 5 patients. ICA was used in 125 (23\%) CAD-RADS 3 patients, 345 (63\%) CADRADS 4 patients and 62 (68\%) CAD-RADS 5 patients (figure 3).

For the diagnostic population, revascularisation was performed in $33(6 \%)$ CAD-RADS 3 patients, $221(40 \%)$ CAD-RADS 4 patients and $46(51 \%)$ CAD-RADS 5 patients (figure 4). Thus, 74\% (92/125) of CAD-RADS 3 patients, $36 \%(124 / 345)$ of CAD-RADS 4 patients, $26 \%(16 / 62)$ of CAD-RADS 5 patients underwent ICA but not revascularisation (figure 5). The majority of CAD-RADS 5 patients undergoing revascularisation were treated with PCI (59\% $(27 / 46))$, and the rest underwent CABG. 


\begin{tabular}{lllllllllll}
\hline Table 2 & Disease severity for 5076 & patients & with diagnostic CTCA at each of the nine sites and overall & \\
\hline Centre & A & B & C & D & E & F & G & H & I & Overall \\
\hline CAD-RADS 0, n (\%) & 388 & 139 & 238 & 75 & 118 & 120 & 195 & 75 & 628 & 1976 \\
& $(40)$ & $(53)$ & $(38)$ & $(39)$ & $(57)$ & $(35)$ & $(39)$ & $(55)$ & $(34)$ & $(39)$ \\
CAD-RADS 1-2, n (\%) & 360 & 67 & 232 & 58 & 60 & 147 & 174 & 37 & 775 & 1910 \\
& $(37)$ & $(25)$ & $(36)$ & $(31)$ & $(29)$ & $(43)$ & $(35)$ & $(27)$ & $(42)$ & $(38)$ \\
CAD-RADS 3-5, n (\%) & 220 & 57 & 163 & 57 & 30 & 74 & 131 & 25 & 433 & 1190 \\
& $(23)$ & $(22)$ & $(26)$ & $(30)$ & $(14)$ & $(22)$ & $(26)$ & $(18)$ & $(24)$ & $(23)$ \\
\hline
\end{tabular}

CAD-RADS allows three distinct groups of patients to be defined: those with normal coronary arteries (CAD-RADS 0), those with atheroma probably not associated with ischaemia (CAD-RADS 1-2) and those with atheroma possibly or probably associated with ischaemia (CADRADS 3-5).

CAD-RADS, Coronary Artery Disease-Reporting and Data System; CTCA, CT coronary angiography.

When ICA was undertaken for CAD-RADS 3 or for a non-diagnostic CTCA, it was chosen in $88 \%(110 / 125)$ and $96 \%(49 / 51)$ of patients, respectively, as the secondline test.

\section{Non-diagnostic CTCA}

Of the 217 (4\%) patients with a non-diagnostic CTCA, $100(46 \%)$ went on to have further investigation. Functional testing for ischaemia and ICA were used equally as a second-line test (49 patients, $49 \%$ for both), and for two $(2 \%)$ patients, ICA was used as a third-line test after functional testing for ischaemia. The non-diagnostic CTCA revascularisation rate (figure 4) was 8\% (18/217) and $65 \%(33 / 51)$ of patients who underwent ICA but not revascularisation (figure 5 ).

\section{DISCUSSION}

This is the second BSCI/BSCCT national initiative aimed at improving CTCA service quality across the UK and follows on from the prospective national radiation dose survey of $2017 .{ }^{7}$ We have shown CTCA to be a robust diagnostic tool with $96 \%$ of scans being diagnostic, across a broad age range of patients, with a mean BMI of $29 \mathrm{~kg}$ / $\mathrm{m}^{2}$ and gender equality in application. Three-quarters

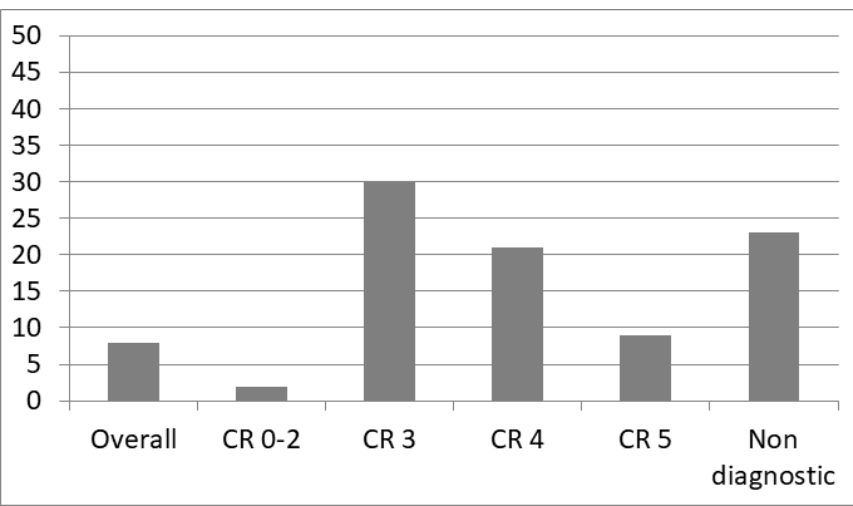

Figure 2 Patients (\%) undergoing functional testing* after all CT coronary angiography by Coronary Artery DiseaseReporting and Data System (CR) score. *Includes patients undergoing functional testing as a third-line investigation after invasive coronary angiography had been used as a second-line investigation. of patients are 'triaged' by CTCA to not require further testing as there is a high probability that their chest pain symptoms are not due to ischaemia. Accordingly. the utilisation of downstream testing in patients from the CAD-RADS 0-2 group was very low, and only 2 of 3883 patients underwent revascularisation with PCI. Nevertheless, a large subgroup of these patients (36\% of the total population) have atheroma and have been identified as potential candidates for preventive therapies based on their CTCA result ${ }^{8}$; this group would not be identified with functional testing for ischaemia.

The total population ICA rate of $12 \%$ appears relatively modest. Furthermore, those patients selected for onward ICA with a CAD-RADS score of either 4 or 5 were likely to undergo revascularisation, at rates of $64 \%$ and $74 \%$, respectively. Of the diagnostic population undergoing ICA, only $12 \%$ underwent CABG. Interestingly, even in the CAD-RADS 5 group, the mode of revascularisation was more commonly PCI ( $59 \%$ PCI vs $41 \%$ CABG). This later finding may be explained by the intrinsic challenges CTCA faces in differentiating between critical stenosis and a short, occluded segment. ${ }^{9}$ This is relevant to procedural planning. Our data support the scheduling patients requiring ICA after CTCA for ICA \pm PCI rather than ICA as a second-line stand-alone diagnostic test.

Despite the clear recommendations in CG95 (2016) that ICA ought to be used as a third-line and not

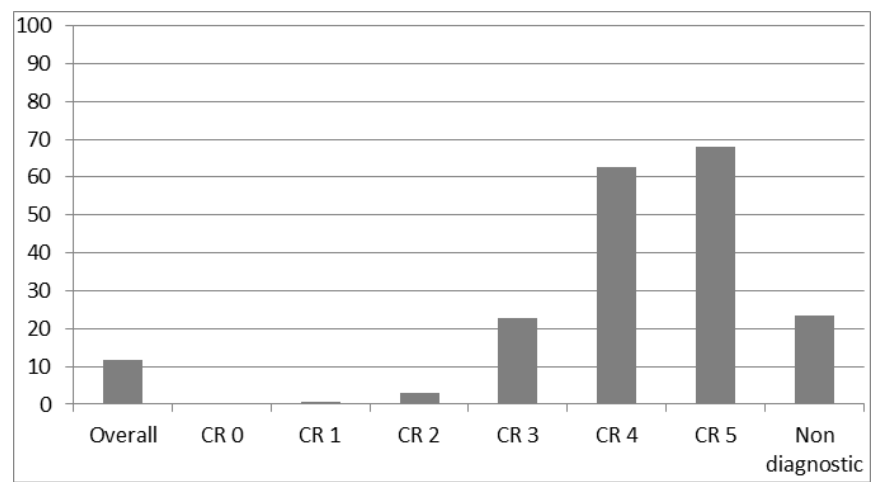

Figure 3 Invasive coronary angiography rates (\%) after all CT coronary angiography by Coronary Artery DiseaseReporting and Data System (CR) score 


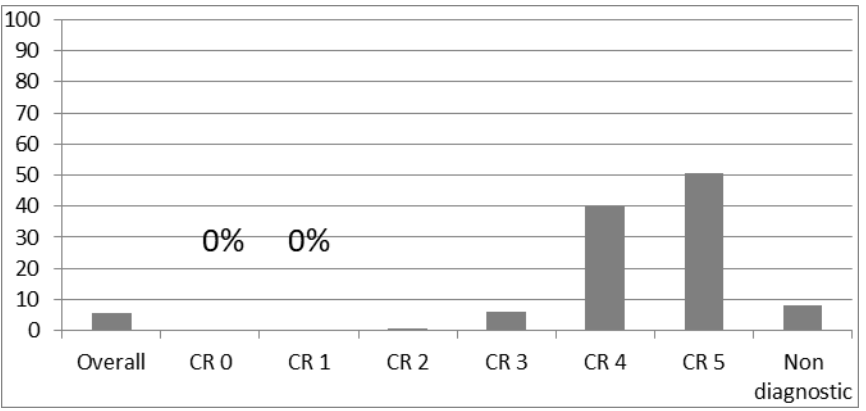

Figure 4 Revascularisation rates after all CT coronary angiography by Coronary Artery Disease-Reporting and Data System (CR) score (\%).

second-line investigation when evidence of $\mathrm{CAD}$ of uncertain functional significance is found or if the CTCA is non-diagnostic, this advice is often not followed. ICA was frequently favoured as the second-line test, and this contributes to the high rate of ICA with no resulting revascularisation $(48 \%)$. The conversely low ICA to PCI conversion rate of $39 \%$, which is consistent with the rate described for the UK by Asher et al in 2019, remains disappointingly low and could be improved. ${ }^{10}$ The 'uncertain functional significance' of CAD-RADS 3 patients is worthy of further attention. Only $6 \%$ of the population with CAD-RADS 3 underwent revascularisation. This suggests that even the recommended 'functional testing for ischaemia when diagnostic doubt remains' (or indeed FFRCT) ought not to be used without serious consideration. It would certainly seem that 'blanket testing' for all CTCA-detected coronary artery stenosis of uncertain functional significance is unnecessary. Indeed, less than $10 \%(46 / 624)$ of patients who underwent either functional testing for ischaemia or FFRCT also underwent revascularisation. Nevertheless, if second-line investigation is indeed thought necessary in either clinical scenario, utilisation of functional testing for ischaemia, rather than ICA, would undoubtedly reduce the rate of duplicated coronary angiographic assessment with no resulting revascularisation.

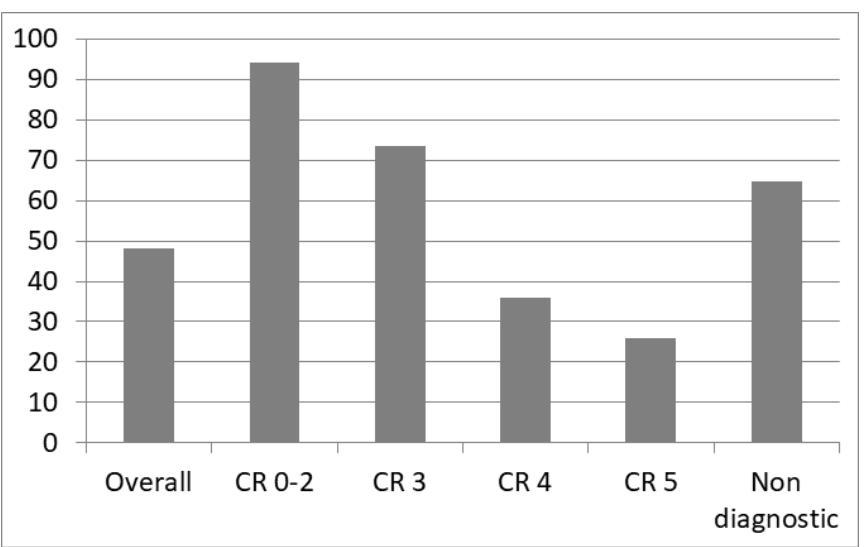

Figure 5 Proportion of patients undergoing invasive coronary angiography without revascularisation (\%) after all CT coronary angiography by Coronary Artery DiseaseReporting and Data System (CR) score.
The data presented in this report may help inform our discussions with patients. The likelihood of a variety of different outcomes is evident at different stages of the patient journey. For example, at the outset of stable recent-onset chest pain investigation, the likely need for follow-on testing after CTCA is low, and the chances of requiring $\mathrm{CABG}$ are very low $(1 \%-2 \%)$. Patients may be listed for ICA because of ongoing clinical concerns and a moderate CTCA stenosis, but there is $74 \%$ chance it will not result in intervention. This report also allows follow-up consultation planning and economic modelling, with $>70 \%$ of patients reasonably dischargeable back to their general practitioner after CTCA without further testing.

Therefore, in terms of service quality improvement, this may be achieved by taking the onward revascularisation rate into account when considering the need for onward testing and then:

- Following NICE CG95 (2016) second-line investigation recommendations.

- Avoiding 'stand-alone' diagnostic ICA after CTCA wherever possible.

These measures may reduce the number of patients undergoing duplicated coronary angiographic assessment with no resulting revascularisation. In turn, this may improve and facilitate the journey of patient with chest pain at less cost and risk.

\section{Strengths and limitations}

Our study provides extensive novel data regarding the contemporary investigation of patients with stable chest pain, although given the timing of data collection the findings may be considered as generally representative of the "pre-FFRCT era'. A major strength of this work is the prospective, multicentre design. The patient numbers are convincing, and the 'real-world' nature of the data allows direct translation to clinical care. It is accepted that the reasons a cardiologist may undertake ICA after a CTCA are heterogeneous and unique to the individual patient. Unclear or suboptimal CTCA reporting may contribute to the decision-making process, and therefore 'ICA with no resulting revascularisation' or 'duplication of coronary angiographic assessment without revascularisation' may be crude as a marker for ICA overuse in this context.

There are of course limitations to a service improvement initiative, which involves collection of data in an observational manner. There may be unadjusted confounding factors and referral bias. The report is intended to provide an overview of the national approach to downstream testing after a NICE CG95 (2016) CTCA, rather than an attempt to identify local geographical variation in patient populations or practice between centres. Every effort has been taken to ensure data collection was accurate and complete, including ensuring that data analysis was reassessed by each contributing centre to ensure accuracy. However, as with all analyses involving healthcare records, these may be incomplete, inaccurate or unclear. One centre did not transfer data with clearly identified CAD-RADS scores and was excluded from 
the analysis. Other centres successfully clarified any unclear or incomplete data entries.

\section{CONCLUSIONS}

CTCA for the NICE CG95 (2016) assessment and diagnosis of recent-onset chest pain has been considered as a robust and effective test. In all, $96 \%$ achieved a diagnostic scan, and of these, $73 \%$ of patients were CAD-RADS 0-2 and therefore should require no further onward investigation. However, $12 \%$ underwent ICA and $48 \%$ of these had duplicated coronary angiographic assessment with no resulting revascularisation. In subgroups (CAD-RADS 3 and non-diagnostic scan patients), there is overuse of ICA as a second-line investigation, suggesting room for improvement in terms of compliance with NICE CG95 (2016). Functional testing for cardiac ischaemia is recommended as second-line investigation for these subgroups although they have been shown to have low overall revascularisation rates $(<10 \%)$, suggesting 'blanket testing' is unnecessary. For patients with severe disease (CADRADS 4 and 5), ICA generally leads to revascularisation (64\% and $74 \%$, respectively), commonly PCI, suggesting a default $\mathrm{ICA} \pm \mathrm{PCI}$ approach is appropriate.

Acknowledgements We thank all the staff who supported this study, including Sylvie Dubois-Marshall, NHS Grampian, Aberdeen, UK; Sze Mun Mak, Guy's and St Thomas' NHS Foundation Trust, London, UK; and Peter Brown, Sheffield teaching hospitals NHS Trust, Sheffield, UK.

Contributors The idea for the study came from GM-H, and the British Society of Cardiac Imaging/British Society of Cardiovascular Computed Tomography supported the study through access to the societal membership. MS, NB, BH, RVL, AV, CAR, ML and GM-H participated in data collection. GM-H, MCW and RB performed analysis or interpretation of the data. ML, CAR, MCW and GM-H drafted the manuscript, and MS, NB, BH, RVL and AV offered critical revision.

Funding The authors have not declared a specific grant for this research from any funding agency in the public, commercial or not-for-profit sectors.

Competing interests None declared.

Patient consent for publication Not required.

Provenance and peer review Not commissioned; internally peer reviewed.

Data availability statement Deidentified participant data are available upon reasonable request to the corresponding author.
Open access This is an open access article distributed in accordance with the Creative Commons Attribution Non Commercial (CC BY-NC 4.0) license, which permits others to distribute, remix, adapt, build upon this work non-commercially, and license their derivative works on different terms, provided the original work is properly cited, appropriate credit is given, any changes made indicated, and the use is non-commercial. See: http://creativecommons.org/licenses/by-nc/4.0/.

ORCID iDs

Gareth Morgan-Hughes http://orcid.org/0000-0001-8568-6440

Michelle Claire Williams http://orcid.org/0000-0003-3556-2428

Margaret Loudon http://orcid.org/0000-0001-7249-2647

\section{REFERENCES}

1 National Institute for health and care excellence (NICE) guidance for the assessment and diagnosis of recent-onset chest pain of suspected cardiac origin (clinical guideline 95 (CG95)).

2 Xie JX, Cury RC, Leipsic J, et al. The coronary artery DiseaseReporting and data system (CAD-RADS): prognostic and clinical implications associated with standardized coronary computed tomography angiography reporting. JACC Cardiovasc Imaging 2018;11:78-89.

3 Abubakr MO, Roobottom CA, Morgan-Hughes GJ. 001 Appropriate use of down-stream invasive coronary angiography (ICA) following computed tomographic coronary angiography (CTCA) is still a work in progress. Heart 2016;102:A1.1-A1.

4 Revised standards for quality improvement reporting excellence (Squire 2.0) September 15, 2015.

5 Cury RC, Abbara S, Achenbach S, et al. CAD-RADS(TM) Coronary Artery Disease - Reporting and Data System. An expert consensus document of the Society of Cardiovascular Computed Tomography (SCCT), the American College of Radiology (ACR) and the North American Society for Cardiovascular Imaging (NASCl). Endorsed by the American College of Cardiology. J Cardiovasc Comput Tomogr 2016;10:269-81.

6 Cury RC, Abbara S, Achenbach S, et al. CAD-RADS ${ }^{\mathrm{TM}}$ : Coronary Artery Disease - Reporting and Data System: An Expert Consensus Document of the Society of Cardiovascular Computed Tomography (SCCT), the American College of Radiology (ACR) and the North American Society for Cardiovascular Imaging (NASCI). Endorsed by the American College of Cardiology. J Am Coll Radiol 2016;13:1458-66.

7 Castellano IA, Nicol ED, Bull RK, et al. A prospective national survey of coronary CT angiography radiation doses in the United Kingdom. $J$ Cardiovasc Comput Tomogr 2017;11:268-73.

8 Adamson PD, Williams MC, Dweck MR, et al. Guiding Therapy by Coronary CT Angiography Improves Outcomes in Patients With Stable Chest Pain. J Am Coll Cardiol 2019;74:2058-70.

9 Hoe J. Ct coronary angiography of chronic total occlusions of the coronary arteries: how to recognize and evaluate and usefulness for planning percutaneous coronary interventions. Int $J$ Cardiovasc Imaging 2009;25 Suppl 1:43-54.

10 Asher A, Ghelani R, Thornton G, et al. Uk perspective on the changing landscape of non-invasive cardiac testing. Open Heart 2019;6:e01186. 